\title{
On the role of response conflicts and stimulus position for hemispheric differences in global/local processing: an ERP study
}

\author{
Gregor Volberg*, Ronald Hübner \\ Universität Konstanz, Fachbereich Psychologie, Fach D29, D-78457 Konstanz, Germany
}

\begin{abstract}
It is widely assumed that the local and global levels of hierarchical stimuli are processed more efficiently in the left and right cerebral hemispheres, respectively. However, corresponding effects were not observed under all circumstances. In ERP studies, they occurred more often with centrally presented stimuli than with laterally presented ones, whereas reaction-time studies revealed that a response conflict between the levels is relevant. The present study examines which of these two factors is more important by presenting conflicting and non-conflicting stimuli to the left or right visual field and recording ERPs as well as collecting behavioral data. If a central stimulus position is crucial, then no effects should show up. Contrary to this prediction, the expected hemispheric differences were observed in the behavioral data as well as in the later occurring (N2 and P3) ERP amplitudes. However, in all variables, the effects were more pronounced for conflicting stimuli. The results suggest that response conflicts are more important for obtaining hemispheric differences in global/local processing than a central stimulus presentation. This is interpreted in the way that hemispheric differences vary with respect to the stimulus representation that is needed to select a proper response.
\end{abstract}

Keywords: Hemispheric difference; Global/local processing; Congruency; Stimulus position

\section{Introduction}

It has widely been assumed that the left and right cerebral hemispheres (LH/RH) are specialized for processing the local and global levels of hierarchically structured visual stimuli, respectively (e.g. Robertson \& Lamb, 1991). For testing this hemispheric specialization compound letters, similar to those depicted in Fig. 1 (cf. Navon, 1977), are often used as stimuli, and the participants have to detect or identify the letter at a pre-specified level. Such stimuli can be applied in combination with several experimental techniques.

For example, in neuropsychological studies it is investigated how unilateral brain lesions affect global and local processing. Their results suggest that damage to the RH impairs the processing of global information, whereas damage to the LH leads to a deterioration of local processing (Delis, Robertson, \& Efron, 1986; Lamb, Robertson, \& Knight, 1990; Robertson, Lamb, \& Knight, 1988). However, unilateral lesions did not always produce the expected neuropsychological impairments. For instance, Polster \& Rapcsak

\footnotetext{
* Corresponding author. Tel.: +49753188 3153; fax: +49 7531883017 .

E-mail address: gregor.volberg@uni-konstanz.de (G. Volberg).
}

(1994) report about two patients who showed normal local processing in spite of massive LH lesions. Also, the results of neuro-imaging studies are inconclusive. Of three studies where positron emission tomography (PET) was used to asses brain activity during global/local processing, only one found effects in the expected direction (Fink, Halligan, Marshall, Frith, Frackowiak, \& Dolan, 1996). A second study failed to replicate this finding (Heinze, Hinrichs, Scholz, Burchert, \& Mangun, 1998), whereas in a third study the pattern of neural activity was actually reversed to that observed in the first one (Fink, Marshall, Halligan, Frith, \& Frackowiak, 1997).

The large variability of these results shows that the conditions under which hemispheric differences for global/local processing occur are still largely unknown. Therefore, the general aim of the present study is to isolate possible favorable factors for this effect. The results should improve our understanding of the mechanisms underlying the hemispheric difference, and could ultimately lead to more precise predictions about the occurrence of hemispheric differences in future investigations.

In the remaining part of this section, we will consider the results of the two most popular experimental techniques. In reaction-time studies, hierarchical letters are either pre- 

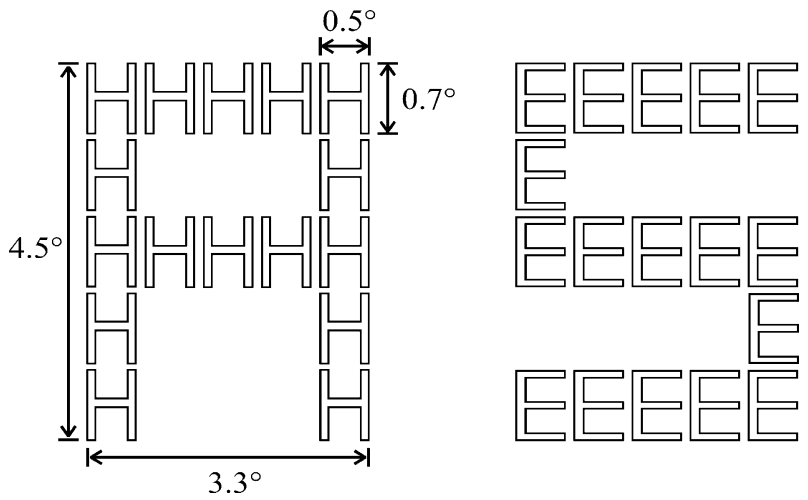

Fig. 1. Two examples of the used stimuli: a global $A$ made from local $H$ 's, and a global $S$ made from local $E$ 's. The size is given in degrees of visual angle.

sented to the left visual field (LVF) or to the right visual field (RVF) and the speed and accuracy of the responses are measured. If the proposed hemispheric specialization exists, then local processing should be faster and more accurate for RVF stimuli, which are initially projected to the $\mathrm{LH}$, than for LVF stimuli projected to the RH. The opposite should hold for global processing. We will call respective differences in reaction times and accuracy VF-effects.

Another method that has often been applied for the investigation of hemispheric specialization is the recording of event-related brain potentials (ERPs), which arise from the synchronous activity of neural populations that are engaged into the actual task. If the hemispheres differ in the proposed way, then it can also be expected that ERP amplitudes recorded from the $\mathrm{LH}$ are larger in the local compared to the global condition, whereas the reverse pattern should occur in the RH. Such amplitude differences will be called hemispheric asymmetries.

Although VF-effects for global/local processing have been reported in several experiments (Blanca, Zalabardo, Garcia-Criado, \& Siles, 1994; Evert \& Kmen, 2003; Hübner, 1997), taken together, reaction-time studies provide not much evidence in favor of hemispheric specialization. This conclusion can also be drawn from two meta-analyses showing that reaction-time studies with positive results were nearly balanced (Van Kleeck, 1989) or even outnumbered (Yovel, Yovel, \& Levy, 2001) by those where no VF-effects were observed. In contrast, the results obtained in ERP studies are more conclusive. Negative results were reported only in a few studies with atypical geometrical figures as stimuli (Han, Fan, Chen, \& Zhuo, 1997; Han, He, Yund, \& Woods, 2001; Johannes, Wieringa, Matzke, \& Münte, 1996). On the other hand, nearly all published ERP studies with compound letters report hemispheric asymmetries in the expected direction (Heinze \& Münte, 1993; Heinze, Johannes, Münte, \& Mangun, 1994 (Experiment 1); Heinze et al., 1998; Malinowski, Hübner, Keil, \& Gruber, 2002; Yamaguchi, Yamagata, \& Kobayashi, 2000).

How can the discrepancy between VF-effects and hemispheric asymmetries be explained? One possible factor that could be responsible for the difference is the stimulus position. In contrast to reaction-time studies, a lateral stimulus presentation is not necessary in ERP studies. Consequently, the stimulus is usually presented to the central visual field (e.g. Heinze et al., 1998; Yamaguchi et al., 2000). That stimulus position might be crucial has also been suggested by Han, Weaver, Murray, Kang, Yund, and Woods (2002). These authors compared ERP studies in which the stimuli were presented centrally with those in which they were presented laterally. If stimulus position matters for the occurrence of hemispheric asymmetries, then the corresponding results should differ. This is indeed the case. Whereas uniform positive results occurred in ERP studies with stimuli in the central visual field (Heinze \& Münte, 1993; Heinze et al., 1994 (Experiment 1); Heinze et al., 1998; Malinowski et al., 2002; Yamaguchi et al., 2000), only two out of four studies with laterally presented stimuli found hemispheric asymmetries (Han, Fan, Chen, \& Zhuo, 1999; Proverbio, Minniti, \& Zani, 1998).

In order to test the effect of stimulus position directly, Han et al. (2002) conducted an experiment in which a compound letter was either presented in the center of the display or in one of the lateral visual fields. As predicted, hemispheric differences showed up only for centrally presented stimuli. The authors explained their result by hypothesizing that the hemispheres compete for the processing of a centrally presented stimulus because both have simultaneous access to the same visual information. This leads to the assignment of more resources to a given target level within the specialized hemisphere. On the other hand, if a stimulus is presented laterally, the hemisphere contralateral to the stimulated hemifield receives the visual information first, whereas the ipsilateral hemisphere receives it only after its transmission through the corpus callosum. The time difference may eliminate the competition between the hemispheres and, accordingly, reduces the associated differences between them (see also Han, Yund, \& Woods, 2003). We will call this account the competition hypothesis.

Although formulated for the explanation of hemispheric asymmetries, the competition hypothesis might also be used to account for the absent VF-effects. That is, lateral stimulation might generally be unfavorable for observing effects indicating hemispheric specializations. This would explain the generally weak and equivocal outcomes obtained in reaction-time experiments. However, there is also evidence that reliable VF-effects can be produced if the information on the stimulus levels is conflicting (for a meta-analysis see Van Kleeck, 1989). It seems that congruent stimuli, i.e. stimuli where both levels activate the same response, are less likely to produce a VF-effect than incongruent stimuli, i.e. stimuli where the levels activate different responses. Supporting results have also been reported in a recent paper by Hübner \& Malinowski (2002). To explain the effect of response conflict on VF-effects these authors proposed that an elaborated stimulus representation is necessary for resolving the conflict induced by incongruent stimuli. They further 
assumed that the hemispheres differ with respect to creating such representations, according to the known specialization. Consequently, hemispheric differences mainly show up in the incongruent situation.

Thus, there are two different factors that have been proposed as being favorable for observing effects of hemispheric specialization. On the one hand, a central stimulus position seems to have a positive effect for hemispheric differences. On the other hand, it has been hypothesized that incongruent stimuli are favorable for VF-effects. However, it seems reasonable to assume that each factor should affect measures obtained in the respective other domain as well. That is, stimulus congruency should also affect hemispheric asymmetries. That this is indeed the case has been shown in an ERP study by Malinowski et al. (2002), where the corresponding effects were most pronounced at temporal-parietal electrodes in the early P3 component $(320-400 \mathrm{~ms}$ after stimulus onset). Since the stimuli were presented to the central visual field and hemispheric asymmetries were nevertheless found only for incongruent stimuli, it follows that a central stimulus presentation cannot be sufficient for obtaining reliable results. Rather, response conflict seems to be the more important factor. This hypothesis would even be further supported by an experiment which shows that a central stimulus presentation is not necessary either.

Such an experiment is reported in the present article. Congruent and incongruent stimuli were either presented to the LVF or RVF and ERPs, reaction times, and error rates were registered. According to the competition hypothesis (Han et al., 2002, 2003) no hemispheric asymmetries should occur under these conditions. Furthermore, if we apply this hypothesis to behavioral data, then VF-effects should also be absent. On the other hand, if congruency is the crucial factor, then hemispheric asymmetries as well as VF-effects should be observed, but only (or at least to a larger extent) for incongruent stimuli.

\section{Method}

\subsection{Subjects}

Sixteen students of the Universität Konstanz participated in the experiment. Some of them fulfilled course requirements; others were paid a small fee for participation. After a preliminary data analysis, four subjects were excluded because of strong artifacts in the EEG data. The remaining subjects (10 females, two males, mean age: 22 years) were right-handed by self-report, had normal or corrected-to-normal vision, and gave informed consent on the experimental procedure.

\subsection{Stimuli}

The stimuli consisted of 16 hierarchical letters which resulted from the pair-wise combination of the four letters $A$,
$S, H$, and $E$ (Navon, 1977, see Fig. 1 for an example). The letters $A$ and $S$ were linked to one response button, whereas the letters $H$ and $E$ were assigned to another one. Therefore, half of the stimuli were congruent and the other half was incongruent. Global letters were constructed from identical local letters in a $5 \times 5$ grid, where the local letters were drawn as white outlines on a black background. At a viewing distance of $81 \mathrm{~cm}$ the local and the global letters subtended $0.5^{\circ} \times 0.7^{\circ}$ and $3.3^{\circ} \times 4.5^{\circ}$ of visual angle, respectively. The LVF and RVF stimuli were presented at an eccentricity of $1.65^{\circ}$, measured from fixation to the midpoint of the stimulus. As cues served the outlines of a red or blue rectangle which signaled the target level by their color. The cues had approximately half the size of the global stimulus form.

\subsection{Apparatus and procedure}

The participants were seated in a sound attenuated chamber in front of a monitor, where a chin rest prevented head movements during the experiment and ensured that the viewing distance remained constant. The stimuli were presented on a 19-in. color monitor with a resolution of 1024 pixel $\times 768$ pixel and a vertical refresh rate of $60 \mathrm{~Hz}$. A trial sequence started with a central $300 \mathrm{~ms}$ presentation of a cue signaling the global or local target level. The cue also served as a fixation. It was stressed in the instructions that it was important to look at the screen center throughout the experiment and to make as few eye movements as possible. After a cue-stimulus-interval of $600 \mathrm{~ms}$, a stimulus appeared for $100 \mathrm{~ms}$ in the LVF or RVF. The task was to categorize the letter at the cued level by pressing an associated button with the index or middle finger of the same hand. Subjects were told to respond as fast and as accurate as possible. As response device served a two-button mouse which was connected to the same computer that also controlled the stimulus presentation. Erroneous responses were signaled by a warning tone. After the response, a black screen was shown for $1300 \mathrm{~ms}$ before the next trail started.

Three factors were varied in the experiment: target level (global and local), visual field (LVF and RVF), and congruency (congruent and incongruent). All factors were randomized. In addition to these experimental factors, the hemisphere of recording ( $\mathrm{LH}$ and $\mathrm{RH}$ ) was included as a factor in the analysis of the ERP data. Response hand, the assignment of letters to response buttons, and the cue color by which the target level was indicated were counterbalanced across participants. The subjects performed 16 blocks with 64 trials within one experimental session of 1.5-2 h. Accordingly, each of the eight experimental conditions had 128 observations per subject.

\subsection{ERP recording}

The continuous EEG was recorded with a 128 channel EGI sensor net (Electrical Geodesics Inc., Eugene, OR) and referenced to the vertex electrode during recording. 
Congruent

Left Hemisphere Right Hemisphere Left Hemisphere
Incongruent

Right Hemisphere

FM

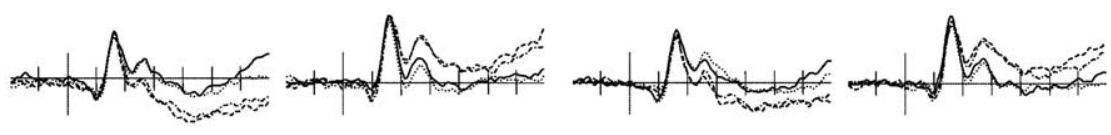

FL

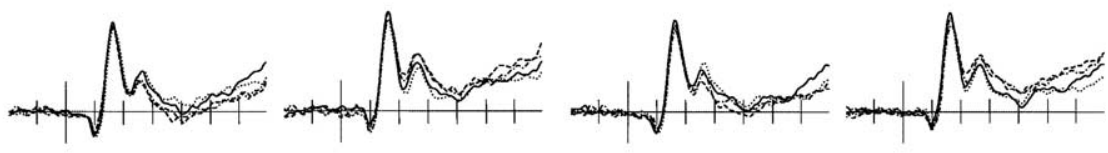

$\mathbf{P}$

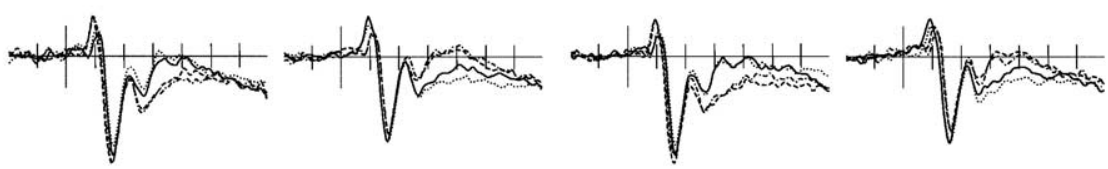

O
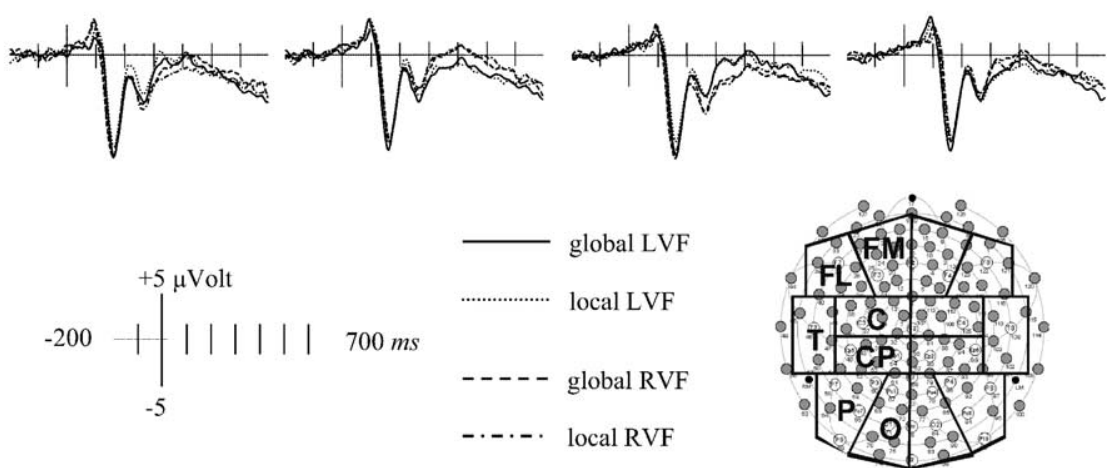

Fig. 2. Grand mean average waveforms at selected electrodes (FM: frontal medial, FL: frontal lateral, P: parietal, O: occipital). The subplots contain waveforms to the global and local levels of left visual field (LVF) and right visual field (RVF) stimuli.

Impedance of the electrodes was kept below $50 \mathrm{k} \Omega$. The EEG was amplified with a bandwidth of $0.1-100 \mathrm{~Hz}$ and digitized at a rate of $250 \mathrm{~Hz}$. After recording, a digital low-pass butterworth filter with $40 \mathrm{~Hz}$ was applied. For artifact correction, the Statistical Correction of Artifacts in Dense Array Studies (SCADS) procedure was used (Junghöfer, Elbert, Tucker, \& Rockstroh, 2000). To this end, the data were arithmetically converted to average reference.

Trials with correct behavioral responses were segmented into epochs of $900 \mathrm{~ms}$, including baseline activity $200 \mathrm{~ms}$ prior to the onset of the stimulus. Neighboring channels were grouped to seven homologous left/right-hemispheric electrode pairs. They are depicted in Fig. 2 (FM: frontal medial, FL: frontal lateral, C: central, T: temporal, CP: centro-parietal, P: parietal, O: occipital). The analysis of the ERP data focused exclusively on the N2 and early P3 components, measured $240-340$ and $340-480 \mathrm{~ms}$ after stimulus onset, respectively. The selections were chosen according to those in earlier relevant studies (Heinze \& Münte, 1993; Malinowski et al., 2002). The ERPs were quantified by the mean amplitudes within the investigated time range and were measured relative to the baseline activity.

\section{Results}

\subsection{Behavioral data}

The data were evaluated by means of repeated-measures analyses of variance (ANOVAs) with latencies of correct responses and error rates as dependent variables. The factors were target level (global and local), visual field (LVF and RVF) and congruency (congruent and incongruent).

For the reaction times there was a significant main effect of target level $[F(1,11)=11.70, P<0.01]$, indicating that the responses to the global level $(635 \mathrm{~ms})$ were faster than those to the local level $(680 \mathrm{~ms})$. Also the factor congruency was reliable $[F(1,11)=56.46, P<0.001]$. Congruent stimuli $(641 \mathrm{~ms})$ produced faster responses than incongruent ones $(673 \mathrm{~ms})$. The latter effect was also mirrored in the error rates $[3.45 \%$ vs. $6.64 \%$ errors, $F(1,11)$ $=11.84, P<0.01]$. Concerning the VF-effects, there was a reliable interaction between target level and visual field in the expected direction [reaction times: $F(1,11)=8.90, P$ $<0.05$; error rates: $F(1,11)=12.76, P<0.01]$. With re- 
spect to the modulation of this interaction by the congruency factor, the results showed a tendency in the expected direction, although the corresponding ANOVAs failed to reach significance [target level $\times$ visual field $\times$ congruency; error rates: $F(1,11)=2.83, P=0.12$; reaction times: $F$ $(1,11)=1.69, P=0.22]$. Nevertheless, if we compute the two-way interaction between visual field and level separately (following Yovel et al., 2001), then it turns out that it was significant only for incongruent stimuli. This was the case for the reaction times, $t(23)=3.16, P<0.005$, as well as for the error rates, $t(23)=3.63, P<0.001$. That is, for the incongruent condition the global level of LVF stimuli was processed faster $(647 \mathrm{~ms})$ and more accurate (5.21\%) than that of RVF stimuli (657 ms, 7.49\%), whereas local processing was accomplished faster and more accurate with RVF stimuli $(680 \mathrm{~ms}, 6.45 \%)$ than with LVF stimuli (709 ms, 7.42\%). On the other hand, the corresponding two-way interactions for congruent stimuli were not significant.

These results are depicted together with the ERP data in Fig. 4a. Each data point represents the reaction time and accuracy of one subject to the global and local levels of LVF stimuli minus those to RVF stimuli. Accordingly, negative differences indicate an LVF advantage, and positive values show a better RVF performance. The colored symbols represent those data where both the latencies and the error rates are in the expected direction. The color value indicates the size of the reaction time difference (see figure legend for details). The figure clearly shows that VF-effects occurred more frequently with incongruent stimuli than with congruent ones. Moreover, the effects were also larger for the former stimuli.

\subsection{ERP data}

The mean ERP amplitudes were subjected to an ANOVA with repeated measures on all factors. The factors were target level (global and local), visual field (LVF and RVF), congruency (congruent and incongruent), and hemisphere of recording ( $\mathrm{LH}$ and $\mathrm{RH})$. Separate analyses were conducted for each electrode pair. The grand mean average ERP waveforms of four representative electrodes are given in Fig. 2. Each subplot contains the responses to local and global targets in the LVF and RVF condition. Fig. 3 shows the difference waves of the local minus global condition in the $\mathrm{LH}$ and $\mathrm{RH}$ for the same electrodes.

\subsubsection{N2 component (240-340 ms)}

Concerning the N2 amplitudes, the analysis showed a main effect for the hemisphere of recording at frontal medial $[F(1,11)=26.55, P<0.001]$, frontal lateral $[F(1$, $11)=12.57, P<0.01]$, and temporal electrodes $[F(1,11)$ $=7.41, P<0.05]$. It showed that the amplitudes were generally larger over the RH compared to the LH.

Furthermore, there was an interaction between the factors visual field and hemisphere of recording at all investigated electrodes $[F(1,11)=17.40-27.23$, all $P<0.01]$. At both frontal electrodes, the amplitudes were stronger in the hemisphere ipsilateral to the stimulated hemifield, compared to contralateral. At the other electrodes the effect was reversed, i.e., the activity was stronger in the hemisphere contralateral to the stimulated hemifield compared to the ipsilateral one.

With respect to hemispheric asymmetries, there was an interaction between the factors target level and hemisphere of recording at both frontal electrodes [FM: $F(1,11)=12.74$,

\section{Congruent Incongruent}

FM
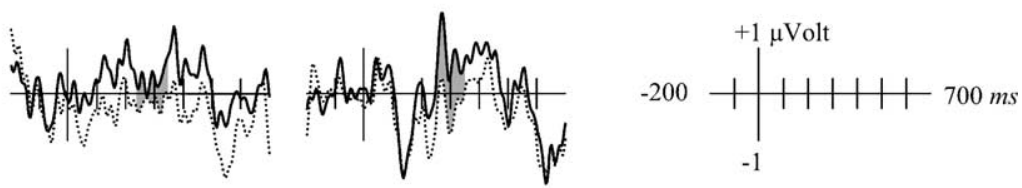

FL
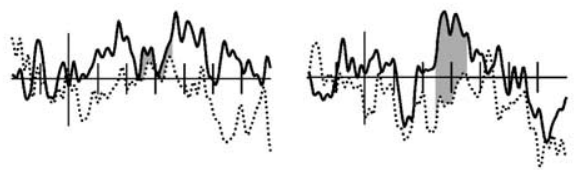

$\mathbf{P}$
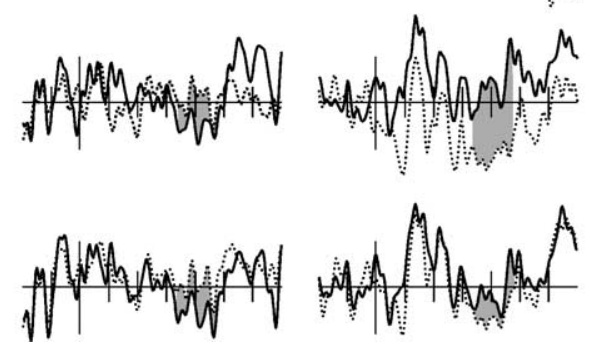

LH (local - global)

RH (local - global)

Fig. 3. Grand mean difference waves of the local minus global conditions in the left hemisphere (LH, solid line) and in the right hemisphere (RH, dotted line). Grey areas mark the time ranges where hemispheric asymmetries were observed. The data show a two-way interaction between hemisphere of recording and target level at frontal medial (FM) and frontal lateral sites (FL), and a three-way interaction between hemisphere of recording, target level and congruency at parietal $(\mathrm{P})$ and occipital $(\mathrm{O})$ electrodes. 
$P<0.01$; FL: $F(1,11)=10.60, P<0.01]$. The data show that in the $\mathrm{LH}$, the amplitudes were larger in the local than in the global condition, whereas in the RH they were larger in the global compared to the local situation (see Fig. 2). Interactions with the factor congruency were not significant. However, when comparing the corresponding difference waves for the congruent and incongruent conditions, one can see that the hemispheric asymmetry was more pronounced in the latter case (see Fig. 3). The difference is largest at frontal lateral sites, where the statistical analysis showed some tendency in that direction [target level $\times$ hemisphere of recording $\times$ congruency, $F(1,11)=1.26, P$ $=0.29$.

\subsubsection{P3 component (340-480 ms)}

The statistical analysis revealed a significant main effect for the factor target level at parietal leads $[F(1,11)=5.06$, $P<0.05]$, indicating that the brain response was larger in the global than in the local condition. A further main effect was obtained for the factor hemisphere of recording. It was

\section{Congruent}

(a)
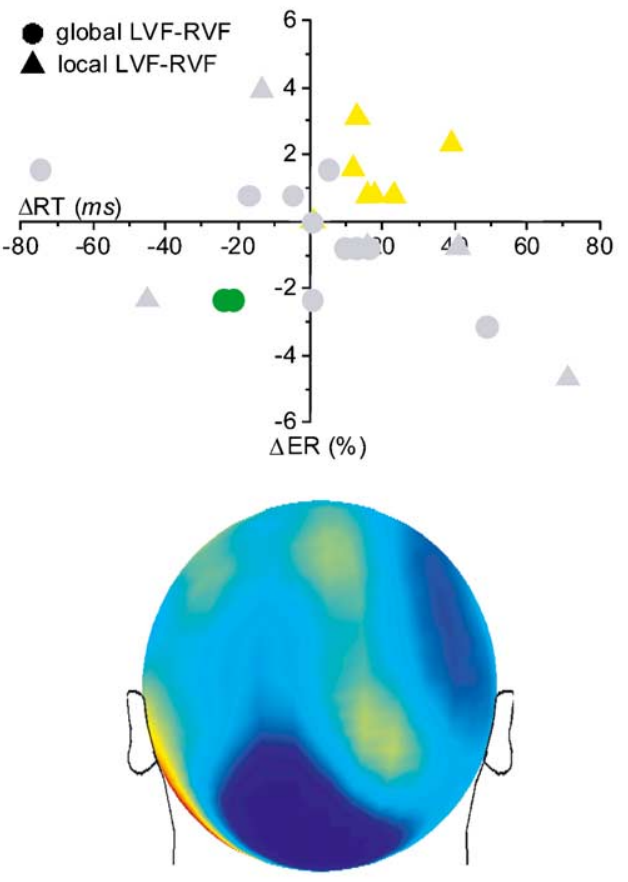

(b)

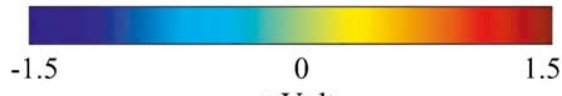

$\mu$ Volt

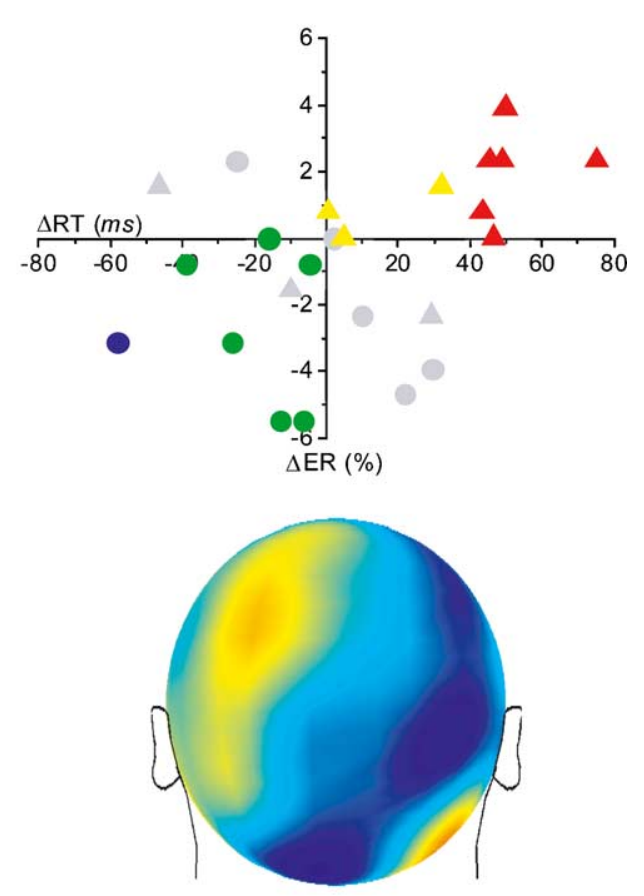

reliable at frontal medial $[F(1,11)=52.63, P<0.001]$, frontal lateral $[F(1,11)=16.31, P<0.01]$ and temporal electrodes $[F(1,11)=9.13, P<0.05]$. The data indicate that the amplitudes were more positive over the right compared to the left hemisphere. Finally, there was a significant main effect for the factor congruency at frontal lateral $[F$ $(1,11)=8.09, P<0.05]$ and centro-parietal electrodes $[F$ $(1,11)=19.81, P<0.001]$. At these sites, the brain activity was larger in the congruent compared to the incongruent situation.

Of the two-way interactions, that between the factors visual field and hemisphere of recording was significant at all investigated electrodes $[F(1,11)=16.74-48.05$, all $P<$ 0.01]. At both frontal leads, the activity was larger in the hemisphere contralateral to the stimulated hemifield compared to that in the ipsilateral one. For the other electrodes this effect was reversed.

Most importantly, the data showed a three-way interaction between the factors target level, hemisphere of recording, and congruency. The effect had a broad posterior distribution

\section{Incongruent}

Fig. 4. (a) Scatter plots showing the responses of every subject to the global (circles) and local (triangles) levels of LVF stimuli minus those to RVF stimuli. Thus, negative values indicate a LVF advantage, and positive values show a RVF advantage. The differences in reaction times ( $\Delta$ RT) and error rates $(\Delta \mathrm{ER})$ are plotted on $x$ - and $y$-axis, respectively. Only colored symbols represent differences supportive for a hemispheric specialization for global and local, whereas grey symbols show that $\Delta \mathrm{RT}$ and/or $\Delta \mathrm{ER}$ were in the unexpected direction. The used color indicates the size of $\Delta \mathrm{RT}$ (blue $=-80$ to $-40 \mathrm{~ms}$, green $=-40$ to $0 \mathrm{~ms}$, yellow $=0$ to $40 \mathrm{~ms}$, red $=40$ to $80 \mathrm{~ms}$ ). (b) Spherical spline-interpolated scalp topographies of the grand mean difference wave, local minus global condition, averaged within the interval $340-480 \mathrm{~ms}$ after stimulus onset. Red color indicates a stronger activity in the local compared to the global condition, and blue color indicates the reversed pattern. One can see that the expected hemispheric asymmetry occurred only for incongruent stimuli. 
that covered adjacent channels at parietal $[F(1,11)=5.93$, $P<0.05]$ and occipital sites $[F(1,11)=5.71, P<0.05]$. Thus, for the further analysis the data of both channel groups were collapsed. The three-way interaction was decomposed by conducting separate analyses for congruent and incongruent stimuli with the factors target level and hemisphere of recording. As expected, the result was significant only in the incongruent condition, $t(47)=1.84, P<0.05$. For congruent stimuli no hemispheric asymmetries showed up, $t$ (47) $=0.11, P>0.40$. This difference can be seen in Fig. $4 \mathrm{~b}$. It shows the scalp topographies of the grand mean difference waves, local minus global condition. Positive values indicate larger amplitudes in the local compared to the global condition, and negative values indicate the opposite pattern. It is evident that the expected local-LH/global-RH specialization was present only for incongruent stimuli.

What is more, when comparing Fig. $4 \mathrm{a}$ and $4 \mathrm{~b}$ one can see that congruency moderated the occurrence of VF-effects and hemispheric asymmetries in the same manner. For incongruent stimuli, local processing was associated with a RVF advantage together with a higher LH activity. At the same time, a LVF advantage occurred in combination with a higher RH activity in the global condition. By contrast, no such effects were observed for congruent stimuli.

Finally, there was a significant four-way interaction between the factors target level, hemisphere of recording, congruency, and visual field $[F(1,11)=10.51, P<0.01]$. It reflects the fact that for congruent stimuli attention to the global level compared to the local level always elicited larger amplitudes in the hemisphere contralateral to the stimulated hemifield [congruent stimuli: target level $\times$ hemisphere of recording $\times$ visual field, $F(1,11)=15.09, P<0.01]$. Consequently, the pattern of hemispheric differences depended on the VF where the stimuli were presented. By contrast, in the incongruent situation it corresponded to the proposed specialization for global/local processing irrespective of the stimulus position [incongruent stimuli: target level $\times$ hemisphere of recording $\times$ visual field, $F(1,11)=0.17, P>$ 0.80]. This difference is depicted in Fig. 5.

\section{Discussion}

From the observation that hemispheric asymmetries occurred more often in ERP studies with centrally presented stimuli than in those with laterally presented stimuli, Han, Weaver, Murray, Kang, Yund, and Woods (2002) concluded that a central stimulus position is favorable for these asymmetries. Such an account could also explain that only some of the behavioral studies found VF-effects for global/local processing, because these studies depend on a lateral stimulus presentation. On the other hand, there is evidence that VF-effects can be produced with incongruent stimuli (Hübner \& Malinowski, 2002; Van Kleeck, 1989). Thus, the
Left Visual Field

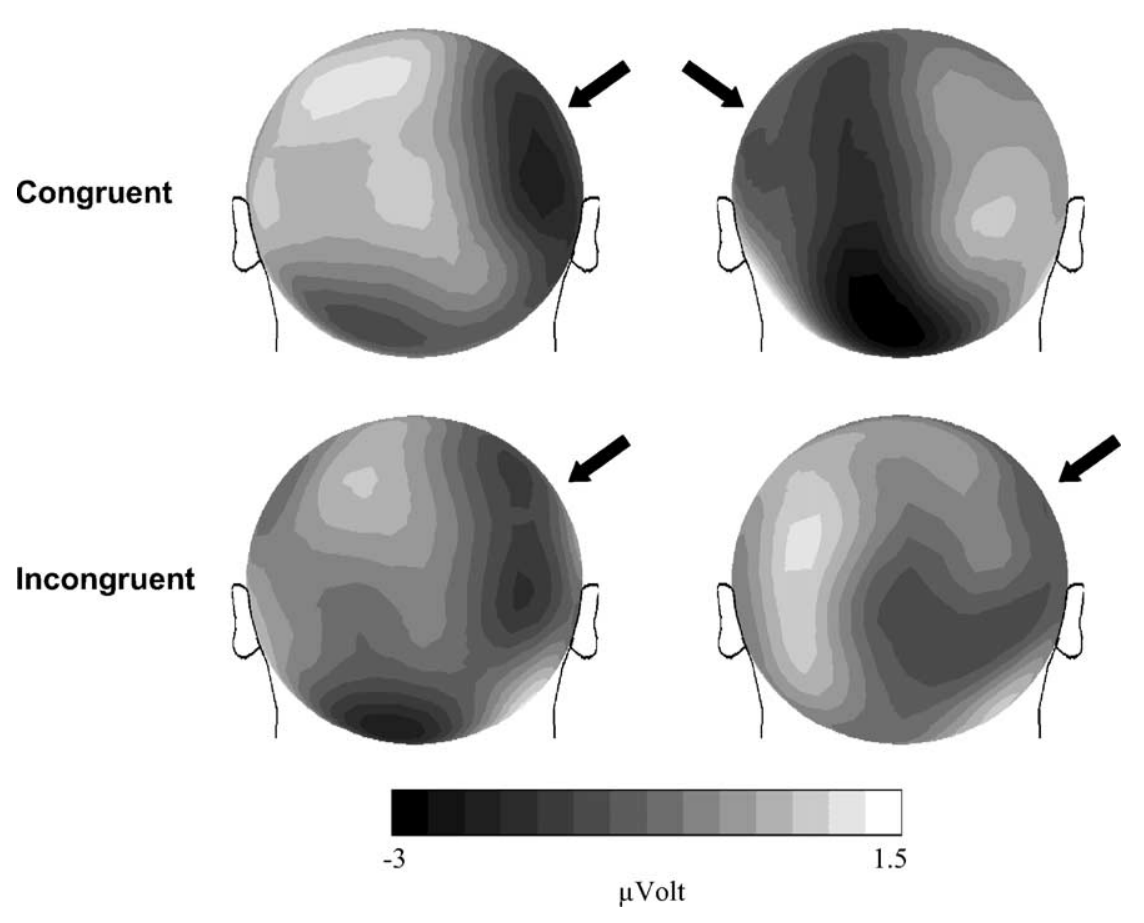

Fig. 5. Spherical spline-interpolated scalp topographies of the grand mean difference wave, local minus global condition (340-480 ms), showing the four-way interaction between the factors target level, hemisphere of recording, congruency, and visual field. The arrows mark that side of the brain where the activity at parietal/occipital sites was larger in the global compared to the local condition. One can see that in the congruent situation, it varied with respect to the hemifield where the stimulus was presented. Contrarily, in the incongruent condition global attention led to a larger RH activity irrespective of the stimulated hemifield. 
question arose whether stimulus position or response conflict is the more important factor for observing the effects of hemispheric specialization. This was tested by presenting congruent or incongruent stimuli either to the LVF or RVF. If a central stimulus presentation is important for obtaining hemispheric asymmetries, then no effects should occur (Han et al., 2002, 2003). However, if the response conflict is important, then hemispheric asymmetries should show up for incongruent stimuli even though they were presented laterally (Hübner \& Malinowski, 2002; Malinowski et al., 2002). Analogous predictions can be made for VF-effects.

Our results clearly show that congruency is the more important factor. First of all, the obtained hemispheric asymmetries occurred in the expected direction. In the $\mathrm{LH}$, the neural activity was larger in response to local compared to global levels, whereas in the $\mathrm{RH}$ the reversed effect showed up. This general pattern of specialization corresponds to the findings of other relevant studies (e.g. Heinze \& Münte, 1993; Malinowski et al., 2002; Yamaguchi et al., 2000). However, the effects were more pronounced for incongruent stimuli. This supports the hypothesis that congruency is important for observing hemispheric differences with respect to global/local processing. A central stimulus presentation is not necessary for obtaining these effects.

Similar results were also observed for the behavioral data. Responses to the global target level were faster and more accurate for stimuli presented in the LVF compared to those presented in the RVF. The opposite was observed for the local target level. These differences, however, were significant only for incongruent stimuli. This demonstrates that both effects are affected in a similar way by congruency. Moreover, to our knowledge the present study is the first where a correspondence was found between VF-effects and hemispheric asymmetries with respect to global/local processing. Of the four published studies which are known to us and where both reaction times and ERPs were recorded, two observed hemispheric asymmetries but no VF-effects (Han et al., 1999; Proverbio et al., 1998). In a third study reliable VF-effects occurred, but there were no hemispheric asymmetries (Heinze et al., 1994 (Experiment 2)). The fourth study found no effects at all (Han et al., 2003).

To provide a mechanism for the modulating effect of congruency, Hübner and Malinowski (2002) proposed an integration hypothesis. They assumed that response selection for congruent stimuli can be accomplished by letter identity alone, and that the hemispheres do not differ with respect to that identification process. However, if one level activates a different response than the other level, also the target level has to be taken into account. In other words, in this case response selection must be based on a stimulus representation where level and letter identity are integrated. It is this integration capacity that is different between the hemispheres, i.e. the $\mathrm{RH}$ and the $\mathrm{LH}$ are specialized for integrating information with the global and local level, respectively. Clearly, the present experiment is not a direct test of the integra- tion hypothesis. However, the obtained results are consistent with this approach.

The integration hypothesis might also explain the observation of Han et al. (2002) that hemispheric asymmetries occurred more often in ERP studies with a central stimulus presentation than in those with a lateral stimulus presentation. In three of the four studies with laterally presented stimuli the target level was blocked (Han et al., 1999, 2003; Proverbio et al., 1998), whereas it was randomized across trials in all ERP-studies with a central stimulus presentation (Heinze \& Münte, 1993; Heinze et al., 1994 (Experiment 1); Heinze et al., 1998; Malinowski et al., 2002; Yamaguchi et al., 2000). It is known that frequent shifts between the target levels are also favorable for the occurrence of hemispheric differences. For instance, in an ERP experiment Heinze et al. (1998) used two different tasks where the target level was either constant throughout a block of trials ('selective attention') or changed in an unpredictable manner ('divided attention'). As a result, the expected hemispheric asymmetries showed up only in the latter condition. Similar positive effects of level randomization were also observed in a reaction-time study by Hübner (1997). It can be assumed that the integration of the target levels with their contents is more difficult when attentional focusing is less efficient due to frequent shifts between the levels. The increased difficulty, in turn, increases hemispheric asymmetries and VF-effects.

A further aspect is that for laterally presented stimuli the relative saliency of the global level is often stronger than that of the local level, whereas for centrally presented stimuli the salience of the levels is usually more balanced (Grice, Canham, \& Boroughs, 1983; Lamb \& Robertson, 1988). It has been shown that the latter case is also favorable for obtaining VF-effects (Yovel et al., 2001). Even this effect could be explained by assuming that the integration process is more difficult the harder it is to discriminate between the levels. If we take the confounding of the stimulus-position factor with other factors into account, then it seems reasonable to assume that a common underlying mechanism explains the effects of stimulus position as well as of response conflict. A promising candidate for such a mechanism is the integration of a stimulus level with its content. In any way, irrespective of which mechanisms finally will account for the observed modulations, the present data show that congruency and the corresponding response conflicts are an important factor for obtaining effects that indicate hemispheric specialization for global/local processing (cf. Hübner \& Malinowski, 2002; Malinowski et al., 2002).

To sum up, our results show that it is important to take the details of the task and the employed stimuli into account. If we had only used congruent stimuli, then we would have observed no VF-effects and hardly any hemispheric asymmetries. Therefore, in order to produce hemispheric differences, more attention should be paid in future studies to the experimental task and to the specific processes that lead to its solution. Such a process-based approach should also be 
helpful for neuropsychologists for predicting possible impairments in global/local processing after unilateral brain damage. Only those tasks should be affected where a more complete stimulus representation is needed for achieving the correct answer.

\section{Acknowledgements}

This research was supported by a grant from the Deutsche Forschungsgemeinschaft (DFG) to the second author as part of a research group (Ro 805/11-1). We thank Franka Glöckner and Anja Nörenberg for the data acquisition, and Andreas Keil for his help concerning the handling of the electrophysiological equipment and the ERP-data analyses.

\section{References}

Blanca, M. J., Zalabardo, C., Garcia-Criado, F., \& Siles, R. (1994). Hemispheric differences in global and local processing dependent on exposure duration. Neuropsychologia, 32, 1343-1351.

Delis, D. C., Robertson, L. C., \& Efron, R. (1986). Hemispheric specialization of memory for visual hierarchical stimuli. Neuropsychologia, 24, 205-214.

Evert, D. L., \& Kmen, M. (2003). Hemispheric asymmetries for global and local processing as a function of stimulus exposure duration. Brain and Cognition, 51, 115-142.

Fink, G. R., Halligan, P. W., Marshall, J. C., Frith, C. D., Frackowiak, R. S., \& Dolan, R. J. (1996). Where in the brain does visual attention select the forest and the trees? Nature, 382, 626-628.

Fink, G. R., Marshall, J. C., Halligan, P. W., Frith, C. D., \& Frackowiak, R. S. J. (1997). Hemispheric specialization for global and local processing: the effect of stimulus category. Proceedings of the Royal Society of London (B), 264, 487-494.

Grice, G. R., Canham, L., \& Boroughs, J. M. (1983). Forest before trees? It depends where you look. Perception and Psychophysics, 33, 121128.

Han, S., Fan, S., Chen, L., \& Zhuo, Y. (1997). On the different processing of wholes and parts: a psychophysiological analysis. Journal of Cognitive Neuroscience, 9, 687-698.

Han, S., Fan, S., Chen, L., \& Zhuo, Y. (1999). Modulation of brain activities by hierarchical processing: a high-density ERP study. Brain Topography, 11, 171-183.

Han, S., He, X., Yund, E. W., \& Woods, D. L. (2001). Attentional selection in the processing of hierarchical patterns: an ERP study. Biological Psychology, 56, 113-130.

Han, S., Weaver, J. A., Murray, S. O., Kang, X., Yund, E. W., \& Woods, D. L. (2002). Hemispheric asymmetry in global/local processing: effects of stimulus position and spatial frequency. Neuroimage, 17, 1290-1299.

Han, S., Yund, E. W., \& Woods, D. L. (2003). An ERP study of the global precedence effect: the role of spatial frequency. Clinical Neurophysiology, 114, 1850-1865.

Heinze, H. J., Hinrichs, M., Scholz, M., Burchert, W., \& Mangun, G. R. (1998). Neural mechanisms of global and local processing: a combined PET and ERP study. Journal of Cognitive Neuroscience, 10, 485-498.
Heinze, H. J., Johannes, S., Münte, T. F., Mangun, G. R. (1994). The order of global- and local-level information processing: electrophysiological evidence for parallel perceptual processes. In H. J. Heinze, T. F. Münte \& G. R. Mangun (Eds.), Cognitive Electrophysiology: Event-related Brain Potentials in Basic and Clinical Research (pp. 102-123). Boston: Birkhauser.

Heinze, H. J., \& Münte, T. F. (1993). Electrophysiological correlates of hierarchical stimulus processing: dissociation between onset and later stages of global and local target processing. Neuropsychologia, 31, 841-852.

Hübner, R. (1997). The effect of spatial frequency on global precedence and hemispheric differences. Perception and Psychophysics, 59, 187201.

Hübner, R., \& Malinowski, P. (2002). The effect of response competition on functional hemispheric asymmetries for global/local processing. Perception and Psychophysics, 64, 1290-1300.

Johannes, S., Wieringa, B. M., Matzke, M., \& Münte, T. F. (1996). Hierarchical visual stimuli: electrophysiological evidence for separate left hemispheric global and local processing mechanisms in humans. Neuroscience Letters, 210, 111-114.

Junghöfer, M., Elbert, T., Tucker, D. M., \& Rockstroh, B. (2000). Statistical control of artifacts in dense array EEG/MEG studies. Psychophysiology, 37, 523-532.

Lamb, M. R., \& Robertson, L. C. (1988). The processing of hierarchical stimuli: effects of retinal locus, locational uncertainty, and stimulus identity. Perception and Psychophysics, 44, 172-181.

Lamb, M. R., Robertson, L. C., \& Knight, R. T. (1990). Component mechanisms underlying the processing of hierarchically organized patterns: inferences from patients with unilateral cortical lesions. Journal of Experimental Psychology: Learning, Memory, and Cognition, 16, 471-483.

Malinowski, P., Hübner, R., Keil, A., \& Gruber, T. (2002). The influence of response competition on cerebral asymmetries for processing hierarchical stimuli revealed by ERP recordings. Experimental Brain Research, 144, 136-139.

Navon, D. (1977). Forest before the trees: the precedence of global features in visual perception. Cognitive Psychology, 9, 353-393.

Polster, M. R., \& Rapcsak, S. Z. (1994). Hierarchical stimuli and hemispheric specialization: two case studies. Cortex, 30, 487-497.

Proverbio, A. M., Minniti, A., \& Zani, A. (1998). Electrophysiological evidence of a perceptual precedence of global vs. local visual information. Brain Research: Cognitive Brain Research, 6, 321-334.

Robertson, L. C., \& Lamb, M. R. (1991). Neuropsychological contributions to theories of part/whole organization. Cognitive Psychology, 23, 299-330.

Robertson, L. C., Lamb, M. R., \& Knight, R. T. (1988). Effects of lesions of temporal-parietal junction on perceptual and attentional processing in humans. The Journal of Neuroscience, 8, 3757-3769.

Van Kleeck, M. H. (1989). Hemispheric differences in global versus local processing of hierarchical visual stimuli by normal subjects: new data and a meta-analysis of previous studies. Neuropsychologia, 27, 11651178.

Yamaguchi, S., Yamagata, S., \& Kobayashi, S. (2000). Cerebral asymmetry of the "top-down" allocation of attention to global and local features. The Journal of Neuroscience, 20, 1-5.

Yovel, G., Yovel, I., \& Levy, J. (2001). Hemispheric asymmetries for global and local visual perception: effects of stimulus and task factors. Journal of Experimental Psychology: Human Perception and Performance, 27, 1369-1385. 